Publicacions Matemàtiques, Vol. 44 (2000), 449-456

\title{
SOME APPLICATIONS OF THE TRACE CONDITION FOR PLURIHARMONIC FUNCTIONS IN $\mathbf{C}^{n}$
}

\author{
Alessandro Perotti
}

\begin{abstract}
In this paper we investigate some applications of the trace condition for pluriharmonic functions on a smooth, bounded domain in $\mathbf{C}^{n}$. This condition, related to the normal component on $\partial D$ of the $\bar{\partial}$-operator, permits us to study the Neumann problem for pluriharmonic functions and the $\bar{\partial}$-problem for $(0,1)$-forms on $D$ with solutions having assigned real part on the boundary.
\end{abstract}

\section{Introduction}

Let $D$ be a smoothly bounded domain in $\mathbf{C}^{n}$. We are interested in some results that can be obtained from the trace condition for pluriharmonic functions introduced by Fichera in the papers $[\mathbf{F} 1],[\mathbf{F} 2],[\mathbf{F} 3]$ and investigated in $[\mathbf{P}]$. This condition has a global character: a real function on $\partial D$ is the trace of a pluriharmonic function on $D$ if it is orthogonal, in the $L^{2}(\partial D)$-norm, with respect to a suitably chosen space of functions. This approach is alternative to the local study of tangential differential conditions (see for example $[\mathbf{R}, \S 18.3]$ and $[\mathbf{F 3}]$ and the references given there).

In this paper we first consider the Neumann problem for pluriharmonic functions. Let $\lambda>0$. Given a real function $\phi$ on the boundary, of class $C^{\lambda}$, we show (Theorem 1) that the solutions of the classical Neumann problem

$$
\frac{\partial U}{\partial \nu}=\phi \quad \text { on } \partial D
$$

are pluriharmonic on $D$ if and only if $\phi$ is orthogonal in $L^{2}(\partial D)$ to the subspace of real harmonic functions that admit a decomposition $H_{1}+i H_{2}$

1991 Mathematics Subject Classification. Primary 32F05; Secondary 32F20, 35N15, $32 \mathrm{D} 15$.

Key words. Pluriharmonic functions, Neumann problem, $\bar{\partial}$-problem.

Partially supported by MURST (Project "Proprietà geometriche delle varietà reali e complesse") and GNSAGA of CNR. 
with $H_{1}, H_{2} \in \operatorname{Harm}_{0}^{1}(D)$ (see $\S \S 2,3$ for the precise definitions). When the domain is the unit ball of $\mathbf{C}^{n}$, this result can be expressed in terms of spherical harmonics. We thus obtain (Corollary 1) another proof of a theorem given by Dzhuraev in [D2]: $U$ is pluriharmonic if and only if it satisfies the Gauss compatibility condition $\int_{S} \phi d \sigma=0$ and the trace condition.

In $\S 4$ we investigate the $\bar{\partial}$-problem for $(0,1)$-forms on $D$ with a boundary condition. Given a $\bar{\partial}$-closed form $f \in C_{0,1}^{0}(\bar{D})$ and a real $C^{\lambda}$ function $g$ on $\partial D$, we look for a solution $u \in C^{1}(\bar{D})$ of the problem

$$
\bar{\partial} u=f \quad \text { on } D, \quad \operatorname{Re} u=g \quad \text { on } \partial D .
$$

We prove (Theorem 2) that a solution exists if and only if $\int_{\partial D} g \bar{\partial}_{n} H d \sigma$ equals the real part of $\int_{D} \bar{f} \wedge * \bar{\partial} H$ for every $H \in \operatorname{Harm}_{0}^{1}(D)$. For this problem too, we can rewrite the compatibility condition on the unit ball in terms of spherical harmonics (Corollary 2). A similar approach to this problem on the ball of $\mathbf{C}^{n}$ appears in [D1].

\section{Notations and preliminaries}

2.1. Let $D=\left\{z \in \mathbf{C}^{n}: \rho(z)<0\right\}$ be a bounded domain in $\mathbf{C}^{n}$ with boundary of class $C^{m}, m \geq 1$. We assume $\rho \in C^{m}$ on $\mathbf{C}^{n}$ and $d \rho \neq 0$ on $\partial D$.

For every $\alpha, 0 \leq \alpha \leq m$, we denote by $P h^{\alpha}(D)$ the space of real pluriharmonic functions of class $C^{\alpha}(\bar{D})$ and similarly for holomorphic functions $A^{\alpha}(D)$ and complex harmonic functions $\operatorname{Harm}^{\alpha}(D)$. We denote by $P h_{\partial D}^{\alpha}(D)$ the space of restrictions to $\partial D$ of pluriharmonic functions in $P h^{\alpha}(D)$ and by $\operatorname{Re} A^{\alpha}(D)$ the space of real parts of element of $A^{\alpha}(D)$.

2.2. For every $F \in C^{1}(\bar{D})$, in a neighbourhood of $\partial D$ we have the decomposition of $\bar{\partial} F$ in the tangential and the normal parts

$$
\bar{\partial} F=\bar{\partial}_{b} F+\bar{\partial}_{n} F \frac{\bar{\partial} \rho}{|\bar{\partial} \rho|}
$$

where $\bar{\partial}_{n} F=\sum_{k} \frac{\partial F}{\partial \bar{\zeta}_{k}} \frac{\partial \rho}{\partial \zeta_{k}} \frac{1}{|\bar{\partial} \rho|}$.

If $\nu$ denotes the outer unit normal to $\partial D$ and $\tau=i \nu$, then we can also write $\bar{\partial}_{n} F=\frac{1}{2}\left(\frac{\partial F}{\partial \nu}+i \frac{\partial F}{\partial \tau}\right)$. The normal part of $\bar{\partial} F$ on $\partial D$ can also be expressed by means of the Hodge $*$-operator and the Lebesgue surface measure $d \sigma: \bar{\partial}_{n} F d \sigma=* \bar{\partial} F_{\left.\right|_{\partial D}}($ see $[\mathbf{K}, \S \S 3.3$ and 14.2]). 
2.3. We shall denote by $\operatorname{Harm}_{0}^{1}(D)$ the real subspace of $\operatorname{Harm}^{1}(D)$

$$
\operatorname{Harm}_{0}^{1}(D)=\left\{H \in \operatorname{Harm}^{1}(D): \bar{\partial}_{n} H \text { is real on } \partial D\right\} .
$$

This space can be characterized in terms of Bochner-Martinelli operator $M$. In $[\mathbf{P}, \S 4]$ it was shown that $F \in \operatorname{Harm}_{0}^{1}(D)$ if and only if $\operatorname{Im} M(F)=\operatorname{Im} F$ in $D$. We recall the integral orthogonality condition that characterizes the traces on $\partial D$ of pluriharmonic functions on $D$ which was introduced (in a different form) by Fichera in $[\mathbf{F 1}]$ (see also $[\mathbf{F 2}],[\mathbf{F 3}])$ and that was studied in $[\mathbf{P}]$ :

$$
\int_{\partial D} U \bar{\partial}_{n} H d \sigma=0
$$

for every $H \in \operatorname{Harm}_{0}^{1}(D)$.

It was shown in $[\mathbf{P}]$ that this condition is necessary for pluriharmonicity when $\partial D$ is of class $C^{1}$ and $U \in C^{\alpha}(\bar{D}), \alpha>0$, or when $\partial D$ is of class $C^{2}$ and $U \in C^{0}(\bar{D})$. The trace condition is sufficient in the case when $U \in C^{1+\lambda}, \lambda>0$. If the boundary value $u$ is only continuous, the same result holds on strongly pseudoconvex domains and on weakly pseudoconvex domains with real analytic boundary.

Remark. When $n=1$ and $H^{1}(D, \mathbf{R})=0$ condition $(\star)$ is void, since $H$ belongs to $\operatorname{Harm}_{0}^{1}(D)$ if and only if $H$ is holomorphic on $D$ (cf. $[\mathbf{P}]$ ).

In general $(n \geq 1)$, the space of $C^{1}(\bar{D})$-holomorphic functions on $D$ is the maximal complex subspace in $\operatorname{Harm}^{1}(D)$ that contains $\operatorname{Harm}_{0}^{1}(D)$. This follows from a theorem of Kytmanov and Aizenberg [KA] (cf. also $[\mathbf{K}, \S \S 14$ and 15$])$ : a $C^{1}(\bar{D})$-harmonic function $F$ is holomorphic on $D$ if and only if $\bar{\partial}_{n} F=0$ on $\partial D$.

2.4. Let $B$ be the unit ball of $\mathbf{C}^{n}$ and let $S=\partial B$. The space $L^{2}(S)$ is the direct sum of pairwise orthogonal spaces $H(s, t), s \geq 0, t \geq 0$, where $H(s, t)$ is the space of harmonic homogeneous polynomials of total degree $s$ in $z$ and total degree $t$ in $\bar{z}$ (see $[\mathbf{R}, \S 12]$ ). These spaces are the eigenspaces of the Bochner-Martinelli operator.

In this case, the trace condition for pluriharmonic functions reduces to the orthogonality to the spaces $H(s, t), s, t>0$. This is the content of a theorem of Nagel and Rudin [NR] (see also $[\mathbf{P}, \S 5]$ ).

\section{The Neumann problem for pluriharmonic functions}

3.1. In this section we study the Neumann problem for pluriharmonic functions: 
Given $\phi$ on the boundary $\partial D$, find necessary and sufficient conditions for the existence of a pluriharmonic function $U$ on $D$ such that $\frac{\partial U}{\partial \nu}=\phi$ on $\partial D$.

We start from the following result announced by Fichera in $[\mathbf{F 1}],[\mathbf{F 2}]$, [F3]. Let $D$ be a simply connected domain, with boundary of class $C^{1+\lambda}$, $\lambda>0$. Let $A, B, C$ be real functions of class $C^{1}(\bar{D})$, harmonic on $D$, such that

$$
\frac{\partial A}{\partial \tau}+\frac{\partial B}{\partial \nu}=0, \quad \frac{\partial A}{\partial \nu}-\frac{\partial C}{\partial \tau}=0
$$

on $\partial D$. Given $\phi \in C^{\lambda}(\partial D)$, there exists a pluriharmonic function $U \in$ $C^{1}(\bar{D})$ such that $\frac{\partial U}{\partial \nu}=\phi$ on $\partial D$ if and only if

$$
\int_{\partial D} \phi(B-C) d \sigma=0
$$

for any triplet $A, B, C$.

The preceding condition is equivalent to the following

$$
\int_{\partial D} \phi\left(H_{1}+i H_{2}\right) d \sigma=0
$$

for every pair $H_{1}, H_{2} \in \operatorname{Harm}_{0}^{1}(D)$ such that $H_{1}+i H_{2}$ is real.

This can be seen setting $H_{1}=-C+i A, H_{2}=-A-i B$. Then $H_{1}, H_{2} \in \operatorname{Harm}_{0}^{1}(D)$ and $H_{1}+i H_{2}=B-C$.

Theorem 1. Let $H^{1}(D, \mathbf{R})=0$ and $\partial D$ of class $C^{1+\lambda}$. Let $\phi \in C^{\lambda}(\partial D)$ be a real function, with $\lambda>0$. Then there exists $U \in P h^{1}(D)$ such that $\frac{\partial U}{\partial \nu}=\phi$ on $\partial D$ if and only if $\phi$ satisfies condition $(\star \star)$.

Proof: We relate the condition $(\star \star)$ to the trace condition $(\star)$. Let $H_{1}$, $H_{2}$ be as before. From the complex version of Green's formula (see for example $[\mathbf{K}, \S 11.3])$ we get

$$
\int_{\partial D} U\left(\bar{\partial}_{n} H_{1}+i \bar{\partial}_{n} H_{2}\right) d \sigma=\int_{\partial D} \partial_{n} U\left(H_{1}+i H_{2}\right) d \sigma
$$

for every real harmonic function $U \in C^{1}(\bar{D})$. Taking real parts, we obtain

$$
\int_{\partial D} U \bar{\partial}_{n} H_{1} d \sigma=\frac{1}{2} \int_{\partial D} \frac{\partial U}{\partial \nu}\left(H_{1}+i H_{2}\right) d \sigma
$$

Now assume that $\phi$ satisfies condition $(\star \star)$. When $H_{1}=1, H_{2}=0$ $(\star \star)$ becomes the Gauss compatibility condition for the classical Neumann problem for harmonic functions. Let $\lambda^{\prime}$ be any positive number smaller than $\lambda$. Let $U \in \operatorname{Harm}^{1+\lambda^{\prime}}(D)$ be real, such that $\frac{\partial U}{\partial \nu}=\phi$ on $\partial D$ (determined up to an additive constant). We show that $U$ satisfies the trace condition. If $H \in \operatorname{Harm}_{0}^{1+\lambda^{\prime}}(D)$, we can set $H_{1}=H$, 
$H_{2}=-\operatorname{Im} H+i G$, where $G \in C^{1}(\bar{D})$ is a real harmonic solution of $\frac{\partial G}{\partial \nu}=\frac{\partial \operatorname{Im} H}{\partial \tau}$ on $\partial D$. Since $H_{1}, H_{2} \in \operatorname{Harm}_{0}^{1}$ and $H_{1}+i H_{2}$ is real, the integral $\int_{\partial D} \phi\left(H_{1}+i H_{2}\right) d \sigma=2 \int_{\partial D} U \bar{\partial}_{n} H d \sigma$ vanishes. From Theorem 2 in $[\mathbf{P}]$ we get that $U$ is the real part of a holomorphic function. Note that in the proof of the cited theorem it is sufficient to consider functions $H \in \operatorname{Harm}_{0}^{1+\lambda^{\prime}}(D)$.

Conversely, if $U$ is pluriharmonic on $D$, then Theorem 1 in $[\mathbf{P}]$ says that $U$ satisfies condition $(\star)$ and then $\int_{\partial D} \phi\left(H_{1}+i H_{2}\right) d \sigma=0$.

Remarks. (i) In effect the proof shows that the condition $(\star \star)$ implies that $U \in \operatorname{Re} A^{1+\lambda^{\prime}}(D)$ even without the topological assumption on $D$.

(ii) If $n=1, H_{1}, H_{2}$ belong to $\operatorname{Harm}_{0}^{1}(D)$ if and only if they are holomorphic functions on $D$. Then $H_{1}+i H_{2}$ is a real valued holomorphic function, that is a constant. As is expected, condition $(\star \star)$ reduces to the Gauss compatibility condition for the Neumann problem.

3.2. If $B$ is the unit ball of $\mathbf{C}^{n}$ and $S$ the unit sphere, the preceeding result can be rewritten in terms of the spaces of harmonic homogeneous polynomials $H(s, t)$. Let $N_{0}$ be the real linear projection in $L^{2}(S)$ introduced in $[\mathbf{P}]$. It is defined for $P_{s, t} \in H(s, t)$ by

$$
N_{0}\left(P_{s, t}\right)=\left\{\begin{array}{ll}
\frac{s}{s+t} P_{s, t}+\frac{t}{s+t} \bar{P}_{s, t}, & \text { for } t>0 \\
P_{s, t}, & \text { for } t=0
\end{array} .\right.
$$

The space $\operatorname{Harm}_{0}^{1}(B)$ coincides with $\operatorname{Fix}\left(N_{0}\right)=\left\{F \in \operatorname{Harm}^{1}(B)\right.$ : $\left.N_{0}(F)=F\right\}$. We show that Theorem 1 in the case of the ball reduces to a result given by Dzhuraev in [D2].

Corollary 1. Let $\phi \in C^{\lambda}(S)$ be a real function, with $\lambda>0$. Then there exists $U \in C^{1}(\bar{B})$, pluriharmonic on $B$ and such that $\frac{\partial U}{\partial \nu}=\phi$ on $S$ if and only if $\int_{S} \phi d \sigma=0$ and $\phi$ is orthogonal to the spaces $H(s, t)$ in $L^{2}(S)$ for any $s, t>0$.

Proof: If $s, t>0$, we set $H_{1}=N_{0}\left(P_{s, t}\right)$ and $H_{2}=N_{0}\left(-\operatorname{Im} H_{1}\right)$. Note that for $s, t>0$ we have $N_{0}(\operatorname{Re} F)=N_{0}(F), N_{0}(\operatorname{Im} F)=N_{0}(-i F)$. An easy computation gives $H_{1}+i H_{2}=\frac{4 s t}{(s+t)^{2}} \operatorname{Re} P_{s, t}$. Replacing $P_{s, t}$ with $i P_{s, t}$, we get $H_{1}+i H_{2}=\frac{4 s t}{(s+t)^{2}} \operatorname{Im} P_{s, t}$. Then the condition $(\star \star)$ is equivalent to the orthogonality of $\phi$ to the spaces $H(s, t)$ and Theorem 1 gives the result. 


\section{4. $\bar{\partial}$-problem with assigned real part on the boundary}

4.1. In this section we study the $\bar{\partial}$-problem for $(0,1)$-forms on $D$ with a boundary condition. Let $f \in C_{0,1}^{0}(\bar{D})$ be a $\bar{\partial}$-closed form with continuous coefficients on $\bar{D}$ and let $g$ be a real continuous function on $\partial D$. We look for a function $u \in C^{1}(\bar{D})$ such that

$$
\bar{\partial} u=f \quad \text { on } D, \quad \operatorname{Re} u=g \quad \text { on } \partial D .
$$

The solution, if it exists, is unique up to an imaginary constant. This problem was considered by Dzhuraev in $[\mathbf{D 1}]$ and $[\mathbf{D 2}]$ in the case of the unit ball.

If $H^{1}(D, \mathbf{R})=0$ and there exists a solution $w$ of $\bar{\partial} w=f$ which is continuous on $\bar{D}$, the problem can be reduced to the trace condition for pluriharmonic functions, since then it amounts to finding a holomorphic function $h$ on $D$ such that $\operatorname{Re}(h+w)=g$ on $\partial D$. If $\partial D$ is of class $C^{2}$ and $D$ is strongly pseudoconvex, there exists a solution operator $S_{q}: C_{0, q}(\bar{D}) \rightarrow C_{0, q-1}(D)$ such that if $\bar{\partial} f=0$ and $f$ is of class $C^{k}(\bar{D})$, then $\bar{\partial} S_{q}(f)=f$ and $S_{q}(f) \in C_{0, q-1}^{k+1 / 2}(\bar{D})$ (see for example [RA] and the references given there). If $\partial D$ is of class $C^{\infty}$ and $D$ is strongly pseudoconvex, another well-known solution operator is given by the Neumann operator $N$ related to $\square$. If $\bar{\partial} f=0$, then $\bar{\partial}^{*} N(f)$ is the unique solution of minimal $L^{2}(D)$-norm of the equation $\bar{\partial} u=f$.

Theorem 2. Let $D$ be a smoothly bounded strongly pseudoconvex domain. Assume that $H^{1}(D, \mathbf{R})=0$. Let $f \in C_{0,1}^{0}(\bar{D})$ and let $g$ be a real $C^{\lambda}$ function on $\partial D(\lambda>0)$. Then there exists $u \in C^{1}(\bar{D})$ such that $\bar{\partial} u=f$ on $D, \operatorname{Re} u=g$ on $\partial D$ if and only if $\bar{\partial} f=0$ and for every $H \in \operatorname{Harm}_{0}^{1}(D)$

$$
\int_{\partial D} g \bar{\partial}_{n} H d \sigma=\operatorname{Re} \int_{D} \bar{f} \wedge * \bar{\partial} H
$$

Proof: Let $\alpha<\lambda$ be a positive number with $\alpha<\frac{1}{2}$. The function $g-$ $\operatorname{Re} S_{1}(f) \in C^{\alpha}(\partial D)$ is the trace of a pluriharmonic function if and only if it satisfies the trace condition $(\star)$. This follows from Theorems 1 and 3 in $[\mathbf{P}]$. For $H \in \operatorname{Harm}_{0}^{1}(D)$, we transform the integral on $\partial D$ involving $f$ in an integral on $D$ :

$\int_{\partial D} \overline{S_{1}(f)} \bar{\partial}_{n} H d \sigma=\int_{\partial D} \overline{S_{1}(f)} * \bar{\partial} H=\int_{D} \overline{\bar{\partial} S_{1}(f)} \wedge * \bar{\partial} H=\int_{D} \bar{f} \wedge * \bar{\partial} H$.

Here we have used the fact that $* \bar{\partial} H$ is a closed $(n-1, n)$-form, since $* \partial(* \bar{\partial} H)=-\bar{\partial}^{*} \bar{\partial} H=-\square H=2 \Delta H=0$. 
The last integral is the Hodge product $(\bar{f}, \partial \bar{H})$ on $D$. Since $\bar{\partial}_{n} H$ is real on $\partial D$, the trace condition becomes

$$
\begin{aligned}
0 & =\int_{\partial D}\left(g-\operatorname{Re} S_{1}(f)\right) \bar{\partial}_{n} H d \sigma \\
& =\int_{\partial D} g \bar{\partial}_{n} H d \sigma-\operatorname{Re} \int_{\partial D} \overline{S_{1}(f)} \bar{\partial}_{n} H d \sigma \\
& =\int_{\partial D} g \bar{\partial}_{n} H d \sigma-\operatorname{Re} \int_{D} \bar{f} \wedge * \bar{\partial} H
\end{aligned}
$$

Note that $P h^{\alpha}(D)=\operatorname{Re} A^{\alpha}(D)$, since the first derivatives of $U \in P h^{\alpha}(D)$ satisfy a Hardy-Littlewood estimate (see $[\mathbf{L}, \S 2]$ for example) and then the same holds for the harmonic conjugate.

4.2. If $D$ is the unit ball, the condition given in the theorem has the following more explicit form.

Corollary 2. There exists $u \in C^{1}(\bar{B})$ such that $\bar{\partial} u=f$ on $B, \operatorname{Re} u=g$ on $S$ if and only if $\bar{\partial} f=0$ and for every $s, t>0$ and $P_{s, t} \in H(s, t)$

$$
\begin{aligned}
& \operatorname{Re} \int_{S} g P_{s, t} d \sigma=\operatorname{Re} \int_{B} \sum_{k=1}^{n} \overline{f_{k}} \frac{\partial}{\partial \bar{z}_{k}}\left(\frac{P_{s, t}}{t}+\frac{\bar{P}_{s, t}}{s}\right) d v \\
& \operatorname{Im} \int_{S} g P_{s, t} d \sigma=\operatorname{Im} \int_{B} \sum_{k=1}^{n} \overline{f_{k}} \frac{\partial}{\partial \bar{z}_{k}}\left(\frac{P_{s, t}}{t}-\frac{\bar{P}_{s, t}}{s}\right) d v .
\end{aligned}
$$

Proof: For $H=N_{0}\left(P_{s, t}\right)$ the left integral in Theorem 2 becomes $\int_{S} g \frac{2 s t}{s+t} \operatorname{Re} P_{s, t} d \sigma$, while the right integrand $\bar{f} \wedge * \bar{\partial} N_{0}\left(P_{s, t}\right)$ is equal to

$$
\sum_{k} \bar{f}_{k} d z_{k} \wedge \frac{2}{s+t}\left(\frac{i}{2}\right)^{n} \sum_{k}(-1)^{k-1} \frac{\partial}{\partial \bar{z}_{k}}\left(s P_{s, t}+t \bar{P}_{s, t}\right) d z[k] \wedge \overline{d z}
$$

Since $d v=\left(\frac{i}{2}\right)^{n} d z \wedge \overline{d z}$, we get the first condition. Replacing $P_{s, t}$ with $i P_{s, t}$ we get the second one.

\section{References}

[D1] A. Dzhuraev, On Riemann-Hilbert boundary problem in several complex variables, Complex Variables Theory Appl. 29(4) (1996), 287-303.

[D2] A. Dzhuraev, On linear boundary value problems in the unit ball of $\mathbf{C}^{n}$, J. Math. Sci. Univ. Tokyo 3(2) (1996), 271-295. 
[F1] G. FicherA, Boundary values of analytic functions of several complex variables, in: "Complex analysis and applications '81 (Varna, 1981)", Bulgar. Acad. Sci., Sofia, 1984, pp. 167-177.

[F2] G. FicherA, Boundary problems for pluriharmonic functions, (Italian), in: "Proceedings of the Conference held in honor of the 80th Anniversary of the Birth of Renato Calapso (Messina/Taormina, 1981)", Veschi, Rome, 1981, pp. 127-152.

[F3] G. Fichera, Boundary value problems for pluriharmonic functions, (Italian), in: "Mathematics today (Luxembourg, 1981)", Gauthier Villars, Paris, 1982, pp. 139-151.

[K] A. M. Kytmanov, "The Bochner-Martinelli integral and its applications", Birkhäuser Verlag, Basel, 1995.

[KA] A. M. Kytmanov and L. A. Aizenberg, The holomorphy of continuous functions that are representable by the BochnerMartinelli integral, (Russian), Izv. Akad. Nauk Armjan. SSR Ser. Mat. 13(2) (1978), 158-169, 173.

[L] E. LigockA, The Hölder duality for harmonic functions, Studia Math. 84(3) (1986), 269-277.

[NR] A. Nagel And W. Rudin, Moebius-invariant function spaces on balls and spheres, Duke Math. J. 43(4) (1976), 841-865.

[P] A. Perotti, Dirichlet problem for pluriharmonic functions of several complex variables, Comm. Partial Differential Equations 24(3-4) (1999), 707-717.

[RA] R. M. RANGE, "Holomorphic functions and integral representations in several complex variables", Graduate Texts in Mathematics 108, Springer-Verlag, New York-Berlin, 1986.

$[\mathrm{R}]$ W. Rudin, "Function theory in the unit ball of $\mathbf{C}^{n}$ ", Grundlehren der Mathematischen Wissenschaften [Fundamental Principles of Mathematical Science] 241, Springer-Verlag, New York-Berlin, 1980.

Dipartimento di Matematica e Applicazioni

Università degli Studi di Milano

Bicocca Via Bicocca degli Arcimboldi 8

I-20126 Milano

Italy

E-mail address: perotti@matapp.unimib.it

Primera versió rebuda el 15 d'octubre de 1999, darrera versió rebuda el 26 de gener de 2000. 